\title{
Utilizing Robotics for Learning English as a Foreign Lan- guage
}

\author{
Dimitris Ziouzios ${ }^{1, *}$, Antonios Chatzisavvas ${ }^{1, * *}$, Akrivi Chaschatzi ${ }^{1, * * *}$, Nikolaos \\ Baras $^{1, * * * *}$, Tharrenos Bratitsis ${ }^{2, \dagger}$, and Minas Dasygenis ${ }^{1, \ddagger}$ \\ ${ }^{1}$ University of Western Macedonia, Department of Electrical and Computer Engineering, Kozani, \\ Greece \\ ${ }^{2}$ University of Western Macedonia, Department of Early Childhood Education, Florina, Greece
}

\begin{abstract}
In recent years, Information and communications technology (ICT) has become a fundamental element of almost all aspects of formal and nonformal education. Educational Robotics (ER) as an ICT subfield has triggered many studies of ER educational utilization as the training of a new foreign language. This paper aims at highlighting another perspective of knowledge, utilizing the ER for learning English as a Foreign Language (EFL). It presents educational scenarios explaining how the robot can be involved in the learning process.
\end{abstract}

\section{Introduction}

Over the last decades, Information and Communication Technologies (ICT) in Education is an interesting field of action for researchers worldwide. Gradually, the research interest has been growing focusing on the effective use of ICT in the learning process on several disciplinary areas. In the age of digital literacy, innovative technological instruments and tools are increasingly available for research within the learning process.

The Robotics field is growing very fast and plays a significant role to most aspects of science and life. Thus, the education education couldn't remain unaffected. Educational Robotics (ER) is used in the classrooms and teachers from all levels of education utilize ER to present novel topics to their students. It is an innovative teaching approach that utilizes programmable systems and it is grounded on project-based learning. It is identified by the use of ICT in the context of the students' abilities for observation, analysis, modeling, and control of various physical tasks [1]. In addition, ER offers opportunities both to educators and children to study STEAM (Science, Technology, Engineering, Arts, Mathematics) fields and other disciplines, such as literacy, through teamwork, problem-solving, and cultivation of collaboration skills and critical thinking [2].

\footnotetext{
*e-mail: dziouzios@uowm.gr

**e-mail: cha.antonis96@gmail.com

***e-mail: akribh.xa@outlook.com

****e-mail: nbaras@uowm.gr

†e-mail: bratitsis@uowm.gr

†e-mail: mdasygenis@uowm.gr
} 
Nowadays, children are very familiarized with computers, tablets and smarthphones, which help them develop skills and understand the world [3]. The researchers often underline that the learning process is about to entirely change, if it follows the trends of the mobile age [4].The whole process of learning and teaching must stays up to date with the demand of the modern way of life to support the trainees' needs [5].Educational technologies, even in early childhood educational contexts [6], play a significant role on education which is transformed from traditional to one that follows more innovative approaches. Regarding technology integration in the context of English as a Foreign Language (EFL), it seems that ICT is very important providing Foreign/ Second language (FL/ L2) trainees with a valuable language experience, enhancing positive experiences and attitudes toward the FL [7].

The paper is structured as follows: initially the theoretical background focuses on learning a foreign language utilizing ICT, , Content and Language Integrated Learning (CLIL) and robotics. Section 3, describes the hardware and software design of an under development robot, the EI-Edurobot. The design and construction process is described, along with the research approach to be followed, through indicative scenarios, as this is an ongoing process at the moment. The paper is finalized with a concluding discussion and ideas to further utilize the proposed EI-Edurobot.

\section{Theoretical Framework}

Learning a foreign language seems to be more easy at a young age, since the positive effects have been long reported to differ in degree, depending on the age of acquisition [8-10].The purpose of teaching English in elementary school is the development of students' language ability to communicate in different ways: linguistic and cultural contexts. Communication therefore has a functional purpose and aims at understanding and producing written and oral speech, depending on the communication context [11]. Its goal is communication, which should be promoted through the acquisition and proper use of vocabulary from the very first stages.

Regarding the use of technological applications in language education, studies conducted in the context of foreign language learning have demonstrated the development of positive attitudes towards learning, reinforcing the learning motivation and students' interest, with the result that the latter achieve higher order thinking skills and better vocabulary retrieval [12]. As a means of information transfer, technological applications facilitate the learning process, encouraging the creation of a collaborative and non-competitive climate, while at the same time helping students to build knowledge in a personal way "combining the new with the old" [13].

An effective educational design would be the well established teaching approache, Content and Language Integrated Learning (CLIL) [14]. The CLIL approach serves as a tool for the promotion of the foreign language teaching and has been praised on many different grounds. First of all, the primacy of meaning over form is considered to have positive effects on the affective dimension, reducing target language anxiety and increasing interest and motivation in the learners [15]. Secondly, it is thought that CLIL is effective, because by being able to comprehend and reason about a content in a foreign language, students have the chance to improve themselves in specific language terminology and generally to expand their cognitive skills (Lasagabaster, 2008). Furthermore, learning about geography, science or history in the CLIL classroom gives the use of the foreign language a purpose and a kind of meaningfulness that is believed to be absent from typical language instruction [15]. This may happen because it provides students with repeated, natural exposure to the language which mirrors the environment of first language acquisition [16]. As a result, learning a foreign language through content provides an opportunity to teach academic tasks and higher order 
thinking skills which is not only beneficial for foreign language students, but also necessary for their overall success in school [16].

Although many students are successful in learning a foreign language regardless the teaching method, it is argued that a wrong combination between the learning style of a student and the teaching style of educator can lead to stress and frustration and not to the achievement of the learning objectives [17]. In order for the students to be more autonomous and the learning outcomes to get improved, it is necessary to examine the use of language learning strategies [17]. O'Malley and Chamot [18], define learning strategies as the special thoughts or behaviors that individuals use to help comprehend, learn, or retain new information. For them, learning strategies are important to language learning because they enhance students' own learning. A similar definition is also given by Oxford [19], defining the learning strategies as "specific actions, behaviours, steps, or techniques - such as seeking interlocutors, or giving to yourself the incentive to deal with a difficult language task - used by students to enhance their own learning". It is believed by many in the field of education that the effective use of learning strategies is one of the most important skills that students must acquire in order to succeed in language learning [20]. When a student deliberately chooses the strategies that match his/her own way of learning, these strategies become a useful tool for active, conscious, and purposeful self-regulation of learning. The learning strategies are important to foreign language learning because they enhance the students' own learning, and students use them for active, self-regulated involvement that is important for the development of communication skills [21]. According to Oxford [19] learning strategies can be classified into six groups: cognitive, metacognitive, memory-related, compensatory, affective, and social. The main focus of the present case study is on the cognitive strategies (which relate to how students think about their learning and enable them to manipulate the language material in direct ways), the memory-related strategies (which relate to how students remember language), the social strategies (which involve learning by interaction with others) and compensatory strategies (which enable students to make up for limited knowledge).

Educational Robotics (ER) firstly appeared in 1980's, in the context of using ICT in the educational process, mainly through the pedagogical trend of the Logo programming language. In general, ER refers to the teaching practice in which students use a robot, usually with the guidance of a teacher, either to develop new knowledge using them or to gain knowledge about the robot itself [22]. The main ER tool is the programmable robot. Thus, ER can be considered as an important instrument for students to build learning experiences [23]. It is related to both the observation and simple handling of a robot as well as more demanding tasks as the students are involved in planning, problem-solving and decision making in relation to the robot's behavior, with the aim of developing creative thinking, the highest level of thinking. The pedagogical objectives of ER are based on two main arguments. The first is based on the preparatory role of school education and concerns ER as a subject of study while the second derives from the pedagogical dimension of education and treats ER as a learning tool [24].

\section{Hardware and Software Design}

\subsection{Hardware Design of the Robotic System}

The robotic system, as depicted in Fig. 1 is designed to be easy to use and secure. The chassis of the robot has been designed with stability and durability as the highest priorities, in order to achieve the optimal weight allocation and center of gravity. As a result, the motion of the robot is smooth and steady. The interior mechanism has been protected by 3D printed covers in order to prevent third parties from accessing the inside components. The interior 
space of the robot has been allocated to the electronic components and structure elements. The robotic system has two arms with double hinges; They are designed in such a way to allow the user to add various components in the robot's hands. The head is able to move in two axes: the horizontal axis (right-left) and the vertical axis (up-down). This design choice allows the robot to express the desired emotions (agreement, denial, irritability, etc.) with higher accuracy. A handle has been added on the top side of the robot to allow easier transfer. Finally, all parts of the robot have been created using polylactic acid or common PLA, as they are biodegradable and bioactive. A key part of the robot is the microprocessor that has

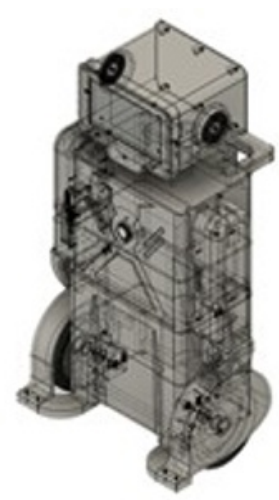

Figure 1. 3D design of the robotic system

been chosen which is the Raspberry Pi 4, an SBC (Single Board Computer). On the board, has been connecting a touchscreen 7-inches monitor, all robot sensors as well as the motors which are in charge of the movement of the hands, head, and wheels.

The main hardware components of the robotic system are shown in Fig. 2. The robot is powered by a $14 \mathrm{~V}$ voltage source with two voltage regulators (Step Down Voltage Regulator). The first voltage regulator outputs $5 \mathrm{~V}$, which supplies all electronic components, the servos, and the microprocessor. The second voltage regulator outputs $12 \mathrm{~V}$ to power the stepper motors. The two stepper motors that the robot has for its movement are controlled by a TB6560 controller each. On one side of the TB6560 driver are connected the external voltage

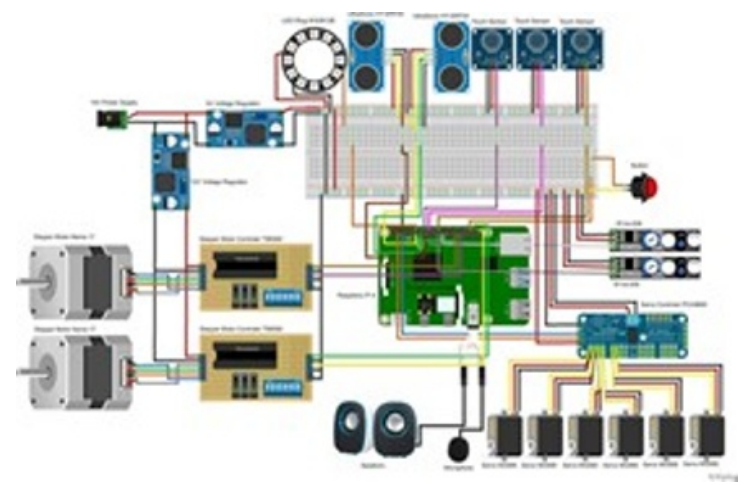

Figure 2. Circuit sketch of the system 
$12 \mathrm{~V}(\mathrm{~V}+)$ for the operation of the stepper motors, as well as it is ground (GND). Then the positive first phase $(\mathrm{A}+)$, the negative first phase $(\mathrm{A}-)$, the positive second phase $(\mathrm{B}+)$ of the stepper motor, and finally the negative second phase (B-) are connected. On the opposite side of the step guide board, there are pairs of positive and negative signals for the communication of the motors and the microprocessor. All the negative parts of the pairs (CLK-, CH-, EN-) are driven to the ground, also the fifth pin $(\mathrm{EN}+)$ is the motor activation signal. The first pin $(\mathrm{CLK}+)$ is the command of the desired steps of the motor and the third pin $(\mathrm{CH}+)$ indicates the direction of rotation of the stepper motor. As for the switches provided by the stepper motor guide board, regulate the maximum current at the input of the motors as well as the settings concerning the step clarity.

The robot has four sensors in order to navigate correctly and safely on the ground. Two of them are responsible for the forward movements and the other two for the backward movements of the robot. At the bottom of the robot, there are two infrared sensors, one on the front and one on the back. Using these sensors, the robot distinguishes when there is ground to move and when not. They have a connection to the microprocessor with one pin each and they have powered by the $5 \mathrm{~V}$ output of the step-down voltage regulator. The robot also has two ultrasonic sensors, which are located one on the front and one on the back to detect obstacles. These sensors are powered by $5 \mathrm{~V}$ from the step-down voltage regulator and are connected to the microprocessor using two pins.

As part of the robot's interaction with users, have used three capacitive touch sensors, a button, and 12 light-emitting diodes (LEDs) in the shape of a ring. The three capacitive touch sensors have the requirement of a $5 \mathrm{~V}$ power supply and a pin to connect them to the microprocessor. The button is a switch with two pins, the first is connected to the $5 \mathrm{~V}$ power supply and the second to the general-purpose pin of the Raspberry Pi 4. Finally, the lightemitting diode (LED) is supplied with $5 \mathrm{~V}$ input voltage and receives commands via a pin from the microprocessor. Users can interract with the robot using the touch screen, the camera and the hands of the robot.

Servo motors are handled by the microprocessor according to the Inter-Integrated Circuit (I2C) communication protocol. This protocol uses two pins, one for data (SDA) and one for clock synchronization (SDL). The PCA9685 PWM controller, which has the responsibility for operating the servomotors, is powered by the $5 \mathrm{~V}$ step-down voltage regulator. Finally, the servo motors are connected to the bottom of the board in a vertical arrangement via three PWM, V++, and GND pins. The robot has been using six servo motors MG995 with an angle of 0-180 degrees.

For the voice and audio communication of the user with the robot, a $3.5 \mathrm{~mm}$ jack microphone was used, and a speaker set with a $3.5 \mathrm{~mm}$ jack input. To connect these two components to the audio input/output port of the board has used a connector (audio splitter).

The 7-inches touch screen is connected to the microprocessor via the DSI (Display Serial Interface) port on the Raspberry Pi 4. The Pi Camera is connected to the microprocessor on the CSI port (Camera Serial Interface), which is also located on the Raspberry Pi 4.

\subsection{Energy requirements}

The robotic system is powered by a $14 \mathrm{~V}-8 \mathrm{Ah}$ lithium battery. The robot has the requirement of lightweight, which provides autonomy as well as short recharging time. The energy requirements of the microprocessor used to amount $3 \mathrm{~A}$. The stepper motors that have the responsibility of moving the robot are powered by $12 \mathrm{~V}$ and consume $2.5 \mathrm{~A}$ each at maximum load. The servo motors that are responsible for the movement of the hands and the head are powered by $5 \mathrm{~V}$ and consume a maximum load of 1.2A. The robot's sensors and indicators consume an average of $400 \mathrm{~mA}$. 


\subsection{Software Design}

The operating system that has been chosen for the microprocessor is Raspberry Pi OS. It is an operating system based on the Debian operating system for Raspberry Pi. The libraries required to detect people and objects are the OpenCV python library and the Caffe model deep learning framework. Installing the PyQt v5 library and QtDesigner was necessary to run the graphical interface as it is depicted in Fig. 5. Pulse audio and Simple audio libraries are responsible for the sound management of the system. The servo motors and the LED ring were operated by Adafruit-circuitPython-servokit and Adafruit-circuitPython-neopixel. The input and output pins (GPIO) were exploited with the pre-installed gpiozero library. The robot is connected to the Internet with ComitUp software that offers the ability to connect to the Internet through another device. Finally, GCompris software was installed to add a variety of educational games to the robotic system.

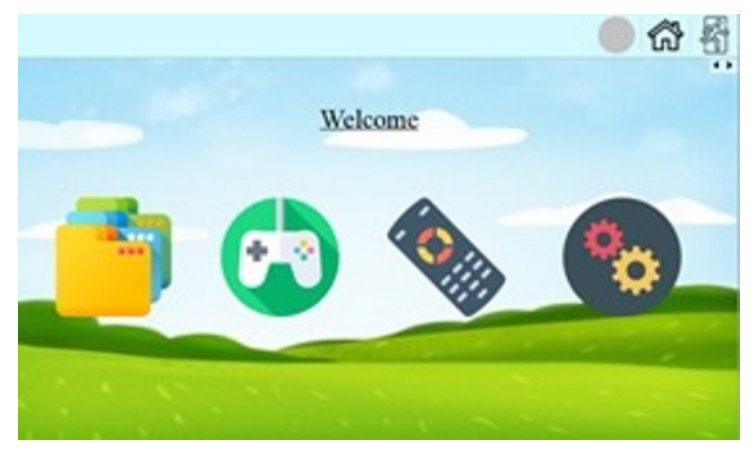

Figure 3. Screenshot of the home page

\subsection{Information System}

The robot can connect with an informatics system, by which the user will be able to run his scenarios. To achieve a secure connection for both parties are required to use a token. This is a piece of data that has no use on its own, but in combination with the right decoding system, is an important piece of security. When the robot starts, it checks for internet connection and only then check for the informatics system connection. After successful control, the robot requests a token, which it uses to subsequently communicate with the system. Tickets for greater security last thirty minutes. At the end of the ticket, the robot requests a ticket again from the informatics system. For the communication of the robotic system with the informatic system and the receipt of a token, a function of the library requests was used. In particular, the function requests.post based on HTTP (Hypertext Transfer Protocol) was used. HTTP is a protocol designed to allow communication between clients and servers, as shown in Fig 3. Acts as a request-response protocol between client and server.

The function RobotCheckConnection(), which is shown in Fig 4, is responsible for controlling the robot's connection to the Internet but also for connecting to the information system. As the first argument, it receives the address of the informatics system. As a second argument receive the name and the serial number of the robot in a JSON type object. In the event of an internet connection, the function sends the JSON object containing the robot name and serial number. If the return status is two hundred, so a successful connection, it receives an object containing the token, which will then be used to communicate with the information system. 
If the situation equals four hundred and one, the name and serial number are not registered in the information system, it responds with an object containing an error message. Finally, in case there is no connection to the informatic system or internet connection it will return the value False.

As already mentioned, the embedded system communicates via WiFi on specified intervals with the IS and transmits all captured images and measurements. Before the transmission process begins, however, all data are being compressed and encrypted. For the captured images, lossless compression formats are being used, such PNG and WebP. For encryption, robots use the asymmetric encryption algorithm RSA and only the IS containing the RSA key can decrypt the data.

The two main components of the proposed IS are the front-end and the back-end. The front-end allows registered users and administrators to access the data regarding the robots. Each robot has a unique identification number transmits encrypted measurements to the server. The back-end of our IS is based on MySQL, an open-source relational database management system and PHP, a general-purpose scripting language.

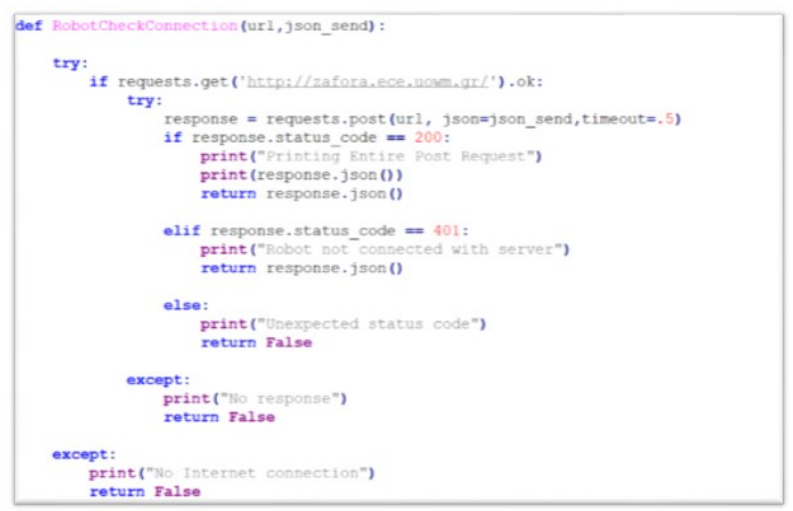

Figure 4. Function RobotCheckConnection

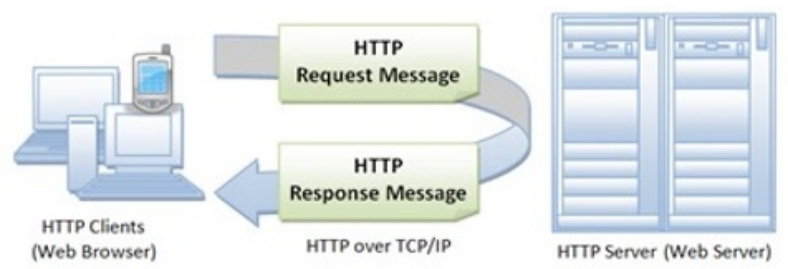

Figure 5. HTTP protocol function

\section{Indicative Scenarios}

This section describes two indicative scenarios concerning the way that the robot can be used for learning English as a foreign language focused on children between 4 and 9 years old. These scenarios were scheduled to be tested until this time but due to the pandemic situation they were postponed. Thus, at this point the scenarios are briefly described. 


\subsection{Giving Directions}

The first scenario is about understanding clearly how to give or take directions. The purpose of this task is to speak in English, giving the right directions to the robot to find the way to a target spot. First of all, the teacher should create a track using for example chairs, playing cubes, pillows etc and distinguish the target by placing an item (e.g. a ball). Of course, other ways of creating a virtual track/maze in a classroom can be followed, based on the available material and space. In any case, one has to reassure that there is enough space for the robot to move around and turn freely. The student must navigate the robot vocally in English in order to reach the designated target. Using the right phrases the robot will executes the directions and the students can will be able to understand his/her mistakes, if any. The robot will be remotely operated by the teacher/researcher, as voice recognition is not implemented yet (but it is an interesting idea to consider for future development). In order to follow the student's directions, the robot operator will either be in the same room or listen to the student through a headset, utilizing the Bluetooth and microphone facility of the robot. He/she would even be able to monitor the movement of the robot through the integrated camera and respond to the student if required (also via Bluetooth). Direction provision is a classic learning activity in all languages as it requires deep understanding of various speech schemas. For example it is included as an activity in the official Greek textbooks, both for the Greek and the English language. The second scenario, cab be divided into has three sub-scenarios.

The main purpose of this scenario is to allow for a free interaction with the robot in the form of a contextualized dialogue, hence making the scenario fall under the CLIL approach. In all cases the student will actually be engaged in a free dialogue through the robot and with the teacher/researcher who will use the Bluetooth based communication facility of the robot.

In the first sub-scenario the dialogue could be related to any topic that the teacher chooses, customizing the scenario at the student's level and needs, but also contextualizing it within the scope of the curriculum followed. For example, the robot can be a local person at a foreign place, a professional or any person that matches the scenario. The student will be required to discuss with the robot in order to receive instructions for finding a place, discuss about pedestrian rules (e.g. the robot acts as a police officer), local cuisine (e.g. the robot acts as a chef), local customs (e.g. the robot acts as an ordinary habitant of another country),

etc. It is obvious that many types of dialogues can be carried out, contextualized in a great variety, as the robot can simulate any type of person. Depending on the case, the robot can even be dressed up accordingly, utilizing cloths or paper cartons.

The second sub-scenario focuses more on cultural and historical issues. In this case the robot will be dressed up (or disguised) as a famous monument. For example it can be the Statue of Liberty or Big Ben (just indicative examples). Then, the student can be engaged in a free form dialogue with the monument, regarding its characteristics, symbolic interpretation, history, etc. This is another example of a CLIL approach which combines EFL and History/Cultural studies. Of course, the robot can undertake any other roles which requires it to simulate an artifact (e.g. a double bus in London) and through that role the goal is to acquire cultural and historical knowledge on the corresponding subject while cultivate also the necessary vocabulary and language skills.

The last sub-scenario involves also a simulation via the robot, but in this case it will concern realistic, everyday situations. For example the robot can play the role of a waiter at a restaurant and the student will be required to place an order for a dinner. Other role ideas could be a hotel front desk employee, a doorman, a tourist/museum guide, a ticket office employee or a retail shop employee. In all these cases the student will be required to initiate a dialogue for requesting information, checking in, issuing a ticket and other everyday activities that a visiting tourist is required to perform in a foreign country. As can be understood, 
the possible scenarios are numerous. The idea behind them is that the child will be less intimidated when attempting to interact with the robot than when doing so with the teacher. The anxiety of making a mistake or even feeling shy or embarrassed to conduct similar dialogues could be significantly less if the activity is perceived by the students as a game with a toy robot.

\section{Discussion-Future Work}

In this paper, the design and development of EI-Edurobot, an educational robot utilized for for teaching English as a Foreign Language to children was presented. Also, indicative scenarios were briefly presented. Using the robot as an intermediate for implementing dialogic activities in order to practice foreign vocabulary and communication in various realistic contexts can further enhance engagement and motivate students.

For the pre-design stage, there were two factors for the development of the EI-Edurobot. The first one was to set children of early childhood and primary education as a target group, as these are the ages at which children learn more easily and built their fundamental social skills and behaviors. The second was the noticeable difference from other ER, as it is open source and open hardware. It was also decided to make freely available all the necessary resources (schematics, list and cost of parts, designed scenarios, programming interface and mobile app) after the completion and keep the required cost much lower than that of the commercially available products. Moreover, we plan to develop a web-based platform which would facilitate the development of a Community of Practice, through which the users will be able to exchange thoughts, solves problems, share codes, exchange lesson plan ideas, etc.

During the 2020-2021 school year, the described scenarios was were planned to be tested in real school settings, but unfortunately due to the current circumstances (pandemic covid-19 based operation of the school, which increases the difficulty by limiting access to schools), it is postponed for the year 2021. So, we prepared to test all of our scenarios in a real classroom and to record students's reactions, levels of knowledge acquisition and how the students feel about being trained by a robot. Finally, we are working on more scenarios that can be used in classroom.

\section{References}

[1] Depover, C., Karsenti, T. and Komis, B. (2010). Teaching using Technology. Athens: Kleidarithmos.

[2] Eguchi,A. (2014). Robotics as a Learning Tool for Educational Transformation. Proceedings of 4th International Workshop Teaching Robotics, Teaching with Robotics and 5th International Conference Robotics in Education.

[3] Yelland, N.J., and Gilbert, C.L.(2013) iPlay, iLearn, iGrow. A report for IBM. Victoria University. Melbourne,

[4] Holden, C. L., and Sykes, J. M. (2011). Leveraging Mobile Games for Place-Based Language Learning. International Journal of Game-Based Learning, 1 (2), 1-18.

[5] Isisag, K., U. (2012). The Positive Effects of Integrating ICT in Foreign Language Teaching, International Conference ICT for Language Learning (5th ed). Florence, Italy, 15-16 November 2012.

[6] Blackwell, C. K., Wartella, E., Lauricella, A. R., and Robb, M. (2015). Technology in the Lives of Educators and Early Childhood Programs: Trends in access, use and Professional Development from 2012 to 2014. Evanston, IL: Center on Media and Human Development at Northwestern University. 
[7] Foley, L. M. (2013). Digital storytelling in primary-grade classrooms (Unpublished PhD. Dissertation). USA: University of Arizona State.

[8] Cummins, J. (1979). Linguistic interdependence and the educational development of bilingual children. Review of Educational Research. https://doi.org.10.3102/00346543049002222

[9] Cummins, J. (1991). Interdependence of first- and second-language proficiency in bilingual children. In E. Bialystok (Ed.), Language processing in bilingual children (pp. 70-89). Cambridge University Press.

[10] Pinter, A. (2017). Teaching Young Language Learners (2nd Ed.). Oxford: Oxford University Press.

[11] Litlewood, W. (1981). Communicative Language Teaching. An introduction. Great Britain: Cambridge University Press

[12] Stepp-Greany, J. (2002). Student perceptions on language learning in a technological environment: Implications for the new millennium. Language, Learning and Technology, 6(1), $165-180$

[13] Solomonidou, Ch. (1999). Educational Technology. Means, materials: didactic use and utilization. Athens: Kastanioti

[14] Dourda, K., Bratitsis, T., Griva, E. and Papadopoulou, P. (2012) 'Combining Game Based Learning with Content and Language Integrated Learning Approaches: A Research Proposal Utilizing QR Codes and Google Earth in a Geography-Based Game'Conference Proceedings, The6th European Conference on Games Based Learning, ed. Dr Patrick F., Waterford Institute of Technology, Cork, Ireland, p.115.

[15] Dalton-Puffer, C. and Smit, U. (2007) "Introduction", In C. Dalton-Puffer and U. Smit (eds.), Empirical Perspectives on CLIL Classroom Discourse, Franktfurt, Vienna: Peter Lang., pp 7-23.

[16] Troncale, N. (2002) "Content-Based Instruction, Cooperative Learning, and CALP Instruction: Addressing the Whole Education of 7-12 ESL Students".

[17] Bull, S and Ma, Y. (2001) Raising learner awareness of language learning strategies in situations of limited recourses. Interactive Learning Environments, 9 (2), 171-200

[18] O’Malley, J.M. and A.U. Chamot. (1990).Learning strategies in Second Language Acquisition. Cambridge: Cambridge University Press.

[19] Oxford, R. L. (2003) "Language learning styles and strategies: An Overview", Heinle and Heinle Thompson International, pp 1-25

[20] Gallo-Crail, R., and Zerwekh, R. (2002) "Language learning and the Internet: Student strategies in vocabulary acquisition", In C. A. Spreen (Ed.), New technologies and language learning: Cases in the less commonly taught languages (Technical Report 25; pp. 55-79), Honolulu, HI: University of Hawai 'i, Second Language Teaching and Curriculum Center.

[21] Oxford, R, (1990). Language learning strategies: What every teacher should knows. University of Alabama. Boston. Heinle and Heinle publications

[22] Misirli, A. and Komis, V. (2014). Robotics and Programming Concepts in Early Childhood Education: A Conceptual Framework for Designing Educational Scenarios. In: Karagiannidis C., Politis P., Karasavvidis I. (eds) Research on e-Learning and ICT in Education, pp 99-118. Springer, New York, NY.

[23] Alimisis, D. (2013). Educational robotics: Open questions and new challenges. Themes in Science and Technology Education, 6(1), 63-71

[24] Fragou, S. (2009). Educational Robotics: Pedagogical Framework and Methodology of interdisciplinary project based learning. In Gregoriadou, M., Gogolou, A., Gouli, E, Gle- 
zou, K., Tsaganou, G, Kanidis, E., Doukakis, D., Fragou, S., and Verginis, E. (Eds.), Teaching Approaches and Tools for Teaching Informatics, pp. 474-490. Athens: New Technology Publications. 